\title{
Development of a Novel Ionic Liquid Based Microemulsion System for Gemcitabine Hydrochloride and in vitro Evaluation in Human Cervical Cancer HeLa Cells
}

\author{
Yuanyuan Chen ${ }^{1}$, Juan $\mathrm{Li}^{2}$,* \\ 'Department of Radiation Oncology, Women's Hospital, School of Medicine, Zhejiang University, Hangzhou, Zhejiang, CHINA. \\ ${ }^{2}$ Department of Radiation Oncology, Affiliated Hangzhou Cancer Hospital, Zhejiang University, School of Medicine, HangZhou, \\ ZheJiang, CHINA.
}

\begin{abstract}
Aim: The aim of this study was to develop novel ionic liquid based microemulsion systems and assessing the in vitro activity against HeLa cancer cell line. Materials and Methods: Four imidazaolium cation based ionic liquids were synthesized and used in the studies. The microemulsions were developed and optimized after studying the phase diagrams comprising isopropyl myristate and different surfactant combinations. The physical characteristics of the prepared formulations were determined by studying globule size, polydispersity index and zeta potential. Also, morphology was studied using transmission electron microscopy. Ex vivo drug release was studied using porcine skin and in vitro evaluation was performed in HeLa cell line. Results: The microemulsions exhibited desirable physical and morphological characteristics. Furthermore, excellent drug release profiles and adequate drug tissue retention were established in ex vivo permeation studies performed using porcine vaginal tissues. The results of cytotoxicity studies revealed that the ionic liquid based microemulsions had noticeably improved potency than conventional w/o microemulsions. Conclusion: lonic liquid based microemulsion systems were prepared for gemcitabine. Although our preliminary evaluation has shown promising results, deeper insights on their therapeutic potential needs to be studies by means of detailed toxicity evaluation and in vivo studies.
\end{abstract}

Key words: Gemcitabine, Microemulsion, Ionic liquid, Cervical cancer, Drug delivery.

\section{INTRODUCTION}

Ionic liquids (ILs) have been historically known as environment-friendly solvents and solubilizes. Owing to their recyclable, non-inflammable and nonvolatile properties, they have found most applications in the field of mechanical, electrolyte and chemical engineering. However, in recent times pharmaceutical researchers have expressed keen interest in using ionic liquids for studying potential pharmaceutical applications. ${ }^{1-3}$ Particularly, ionic liquid based microemulsions (hereinafter, IL-microemulsions) have gained considerable upsurge primarily due to excellent solubilizing ability and tunable physical characteristics, which make them ideal carrier for both hydrophilic as well as hydrophobic drugs. ${ }^{4,5}$

The present study aims to develop and evaluate a novel IL-microemulsion using gemcitabine hydrochloride $(\mathrm{GMH})$ as a model anticancer drug. GMH, commonly known as Gemzar, is a low-molecular weight drug indicated for pancreatic cancer, breast cancer, ovarian cancer, non-small cell lung cancer and cervical cancer. Despite the wide spectrum of anticancer efficacy of GMH, it is associated with a major drawback, i.e., extremely short half-life of few minutes. To counter this, the drug is administered
Submission Date: 09-05-2020; Revision Date: 02-12-2020; Accepted Date: 18-06-2021

DOI: 10.5530/ijper.55.3.140 Correspondence: Dr. Juan $L i$

Department of Radiation Oncology, Affiliated Hangzhou Cancer Hospital, Zhejiang University, School of Medicine, HangZhou, ZheJiang, CHINA. Phone no: 0086-057156006302

Email id: KendraOneillybq@ yahoo.com

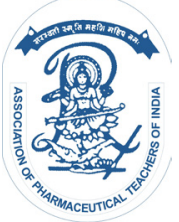

www.ijper.org 
in high doses, which results in many serious adverse effects like bone marrow suppression, liver and kidney toxicity, neuropathy and hair loss. ${ }^{6,7}$ Therefore, many researchers have attempted in past to develop a variety of drug delivery systems for GMH for improving patient compliance by reducing the drug dose and thereby, the associated adverse effects. Out of the studied drug delivery systems, microemulsion system is most practical pharmaceutical carrier due to ease of manufacture, excellent physical stability and costadvantage.

Microemulsions are thermodynamically stable, monoisotropic pharmaceutical carrier systems, which comprise of a hydrophilic, a hydrophobic and an amphiphilic phase, which in conventional sense mean water, oil and surfactant, respectively. However, for IL-microemulsions, the ionic liquids are used as hydrophilic or hydrophobic phases. Our experimentation included synthesizing several ionic liquids and screening appropriate ionic liquid to be used as drug reservoirs for loading GMH. The selected microemulsions were characterized with various analytical methods and the in vitro anticancer efficacy was observed in human HeLa cells. We believe that the IL-microemulsions could provide excellent alternative to the conventional injectable therapies for a variety of cancers, such as pancreatic cancer, breast cancer, ovarian cancer, non-small cell lung cancer and cervical cancer. Additionally, the developed IL-microemulsions are specifically designed for topical administration to address the patient compliance and medication related issues of already marketed Gemcitabine injectable dosage forms.

\section{MATERIALS AND METHODS}

Gemcitabine was kindly provided by Sinopharma Pvt. Ltd as a gift sample. The polyoxyethylene sorbitan ester (Tween 80), sorbitan ester (Span 20) and isopropyl myristate oil was purchased from Jiangsu Hengrui Medicine Co., Ltd.1-methylimidazole, 4 n-alkyl halides (bromoethane, bromopropane, bromobutane and bromopenatne) and 3-(4,5-dimethylthiazol-2-yl)5-(3-carboxymethoxyphenyl)-2-(4-sulphophenyl)-2Htetrazolium (MTS)were procured from Sigma Aldrich Co., USA. All other reagents used were purchased from local market and were of analytical grade. The vaginal tissues of adult female pigs (about one year old) were collected for ex vivo evaluation of drug release and retention from a local slaughterhouse in Hangzhou. The human HeLa cell line was procured from Shanghai
Institute of Cell Biology, Chinese Academy of Sciences (Shanghai, China).

\section{Synthesis and analysis of ionic liquids}

The ionic liquids used in the study were synthesized using previously reported schemes. ${ }^{8,9}$ Briefly, 1-methylimidazole (10 mmoles) was refluxed with $n$-alkyl halide(s)(12 mmoles) in acetonitrile $(25 \mathrm{~mL})$ till complete conversion of starting material to product (Figure 1). The reaction progress was monitored using thin layer chromatography on pre-coated silica gel aluminium plates. The finished products were afforded after evaporating the unused alkyl halide using flash rotary evaporator (Rotavapor R-300, Buchi India Ltd). The isolated products were characterized by Nuclear Magnetic Resonance (NMR) spectroscopy (Ultrashield-400, Bruker USA Ltd.) and mass spectroscopy (Finnigan MAT LCQ, Thermo Electron Corp., USA).

\section{Solubility study}

The saturation solubility of GMH was determined in various ionic liquids using previously established shaking flask method. Briefly, excess amount of the drug was shaken in a solubility shaker (Incubating orbital shaker, VWR UK Ltd.) for $48 \mathrm{~h}$ in capped 25 $\mathrm{mL}$ conical flasks at a temperature of $37^{\circ} \mathrm{C}$. Thereafter, the supernatants were filtered and analyzed by UV spectroscopy (UV1601, Shimadzu Corp., Japan)

\section{Preparation and characterization of microemulsions}

Pseudo-ternary phase diagrams were constructed to identify the microemulsion forming concentrations of the three phases, i.e., isopropyl myristate (oil phase), ionic liquid (hydrophilic phase) and Tween 80 and Span 20 mixture in 2:1 w/w ratio (amphiphilic phase). The Tween 80 and Span 20 mixture (hereinafter, SMix) was selected on the basis of their ability to emulsify ionic liquids in isopropyl myristate oil as previous reported in literature. ${ }^{10}$ The ionic liquids were drop wise

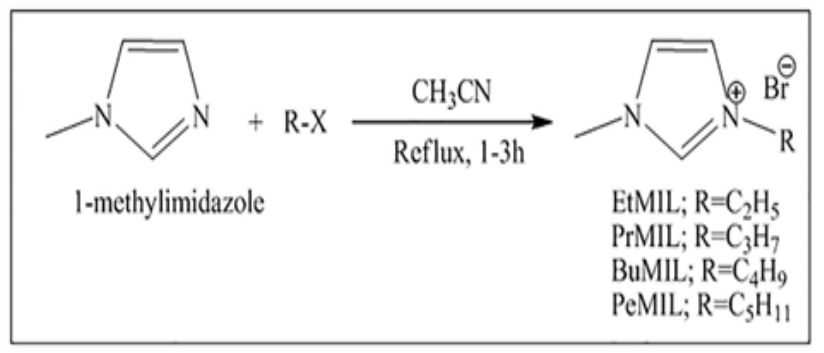

Figure 1: Schematic representation of synthesis of ionic liquids. 
added to vigorously shaken oil and SMix combinations prepared in different ratios ranging from 1:9 to 9:1 w/w. The isotropic clear fluid mixtures were characterized as microemulsions, whereas the translucent fluid mixtures were characterized as emulsions. The microemulsion regions from the pseudo-ternary phase diagrams were utilized to select the microemulsion compositions.

The microemulsion formulations were prepared using simple vortex mixing. The oil and SMix in required amounts were placed in a glass vial and were stirred until consistent. GMH was solubilised in the required amount of ionic liquid and thereafter, the ionic liquid, was added drop wise with continuous shaking. Transparent drug loaded IL-microemulsions were obtained using the synthesized ionic liquids. The respective blank IL-microemulsions were prepared with plane ionic liquids by using similar procedure. Also, for comparison, a drug loaded w/o microemulsion was prepared using a previously reported method. ${ }^{11}$

The mean globule size, polydispersity index and zeta potential were studied using Zetasizer (3000HS, Malvern Instruments Ltd., UK) at $25^{\circ} \mathrm{C}$ using a scattering angle of $90^{\circ}$ (size measurements). Also, globule morphology of the selected IL-microemulsion was determined using transmission electron microscopy (hereinafter, TEM) (T-7000, Hitachi Ltd., Japan).

\section{Ex vivo vaginal permeation and retention studies}

A Franz diffusion cell assembly platform (Permegear Inc., USA) was used to perform the ex vivo release study of the selected IL-microemulsion in porcine vaginal mucosa. The vaginal tissue was cautiously separated from the underneath layers, cleansed with normal saline $(0.9 \% \mathrm{NaCl}$ solution)and cut into smaller squares (about $3 \times 3 \mathrm{~cm}$ ) and were used fresh. The formulation $(200 \mu \mathrm{l})$ was placed on the vaginal tissue fixed in the Franz donor compartment with a positive-displacement micropipette. Each Franz acceptor compartment was filled with $30 \mathrm{~mL}$ of phosphate buffer saline (PBS), $\mathrm{pH}$ 7.5 , which was kept at $37 \pm 1^{\circ} \mathrm{C}$ and constantly stirred $(100 \mathrm{rpm})$. At predetermined intervals, an aliquot of 2 $\mathrm{mL}$ sample was withdrawn and replaced immediately with an equal volume of PBS sink medium. The samples were analyzed using HPLC method. After 6 $h$, the vaginal tissue pieces were removed from Franz cell assembly and carefully wiped with wet cotton. The tissues were homogenized in methanol and quantified for drug by HPLC.

\section{In vitro cytotoxicity in human HeLa cell line}

The human HeLa cell line were procured and lymphocyte isolation was performed using gradient separation medium (Lymphocyte $\left.{ }^{\circledR}-\mathrm{H}\right)$. Cells were maintained in EX-CELL medium, comprising penicillin $(100 \mathrm{U} / \mathrm{mL})$ and streptomycin $(100 \mathrm{mg} / \mathrm{mL})$. The MTS assay was performed using reported methods. ${ }^{12,13}$ For performing the MTS assay, cells were seeded in a concentration of $5 \times 10^{4}$ cells per well in 96 well plates and thereafter IL-microemulsions were incorporated in the wells. Following $48 \mathrm{~h}$ of inoculation, Cell Titer $96^{\circledR}$ Aqueous One Solution Reagent (Promega, USA), was included in every well. Finally, cell viability was analyzed by studying the absorbance values $(490 \mathrm{~nm})$ with ELISA microplate reader (ACTGene, Inc., USA)

\section{Statistical analysis}

All results represent mean $\pm \mathrm{SD}$ from experiments preformed in triplicate parallel runs. Statistical comparison was performed using One-way analysis of variance (ANOVA) with Tukey's test. The $p$ values smaller than 0.05 were considered statistically significant.

\section{RESULTS AND DISCUSSION}

The ionic liquids were synthesized and afforded after evaporating the unused alkyl halides. The characterization of the isolated compounds was performed using ${ }^{1} \mathrm{H}$ NMR and MS spectroscopy (data not shown).

The first step of the study was to analyse the solubility of GMH in the prepared ionic liquids (Figure 2). The results indicated that synthesized ionic liquids exhibit exceptional solubility for GMH. Particularly, EtMIL, PrMIL and BuMIL show 3.8, 4.5 and 2.8 times enhancement of drug solubility, respectively in comparison to that in water. The remarkable enhancement of drug solubility could be attributed to solute-solvent interactions such as Van der Waal's

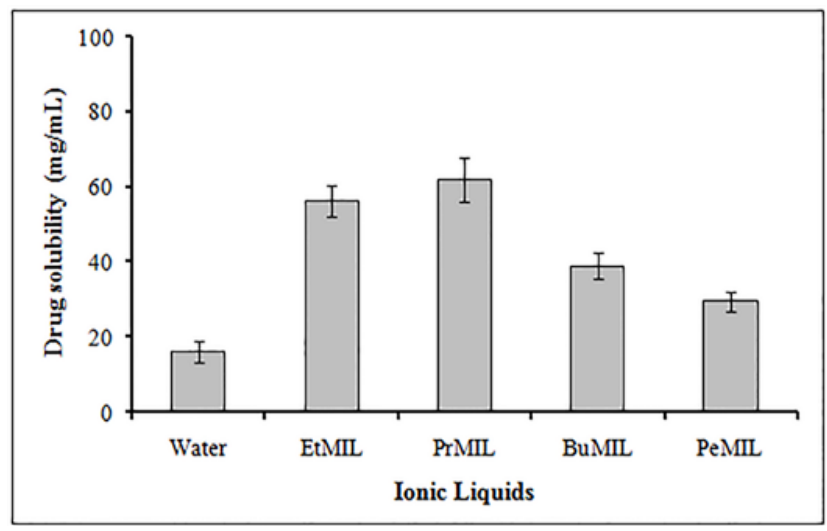

Figure 2: Solubility of GMH in the synthesized ionic liquids. 
interactions, $\mathrm{H}$-bonding and $\pi-\pi$ interactions between aromatic rings of imidazolium cation and GMH.

The pseudoternary diagrams for the prepared ionic liquids have been shown in Figure 3. All ionic liquids exhibited excellent microemulsion region, a feature that ensures adequate microemulsion forming combinations. Generally, points selected from inner microemulsion regions provide isotopic and stable microemulsions, while those selected from region near interface line provide potentially unstable microemulsions. Therefore, we selected the microemulsion compositions from inner microemulsion regions (Table 1).

Also, the studied various microemulsion characteristics have been provided in Table 1. Globule diameter size is considered an important parameter as it affects microemulsion stability, topical permeation and hence ultimately in vivo efficacy. The prepared IL-microemulsions exhibited mean globule diameters in range of $164 \mathrm{~nm}$ to $188 \mathrm{~nm}$, i.e., well below the desired value $300 \mathrm{~nm}$ (Figure 4). Globule sizes below this value are considered optimum topical drug delivery. ${ }^{14,15}$ The small values of mean diameter could be attributed to lower fluidity and surface viscosity of the interfacial film for IL-micromeulsions, which reduce the radius of curvature of the nanodroplets and form transparent microemulsionsystems. ${ }^{10}$ The spherical morphology of IL-microemulsions was observed by transmission electron micrographs, which was in consonance with the previous reports (Figure 5). Also, the mean polydispersity indices of the microemulsions ranged from 0.15 to 0.24 , approximately, which indicates adequate microemulsion stability and shelf life. ${ }^{16}$ The zeta potential values of IL-microemulsions were found to be 9.7 to $12.8 \mathrm{mV}$. The microemulsions with positive zeta potential have been reported to exhibit higher topical drug retention values because of negative charged phospholipids moieties in mucosal linings. ${ }^{17,18}$ Therefore, the prepared ionic liquids are extremely suitable for topical drug delivery in vaginal mucosa.
Finally, the IL-microemulsions exhibited desired viscosity at $37^{\circ} \mathrm{C}$ ranging from 34.33 to $51.67 \mathrm{cpsx} 10^{3}$. Also, the viscosity was not affected significantly ( $p>0.05)$ after drug loading. The viscosity characteristics were favorable to ensure prolonged application of the microemulsions on topical surfaces.

It was observed that the IL-microemulsions upon maintaining for 6 months at ambient storage

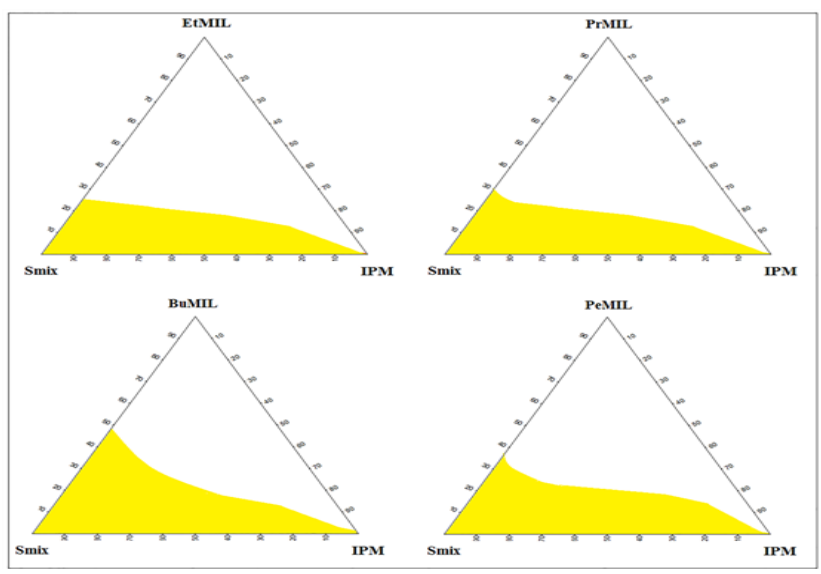

Figure 3: Pseudoternary phase diagrams constructed with IPM, ionic liquids and Smix (Tween 80 and Span 20, 2:1 w/w); The shaded area represents microemulsion region.

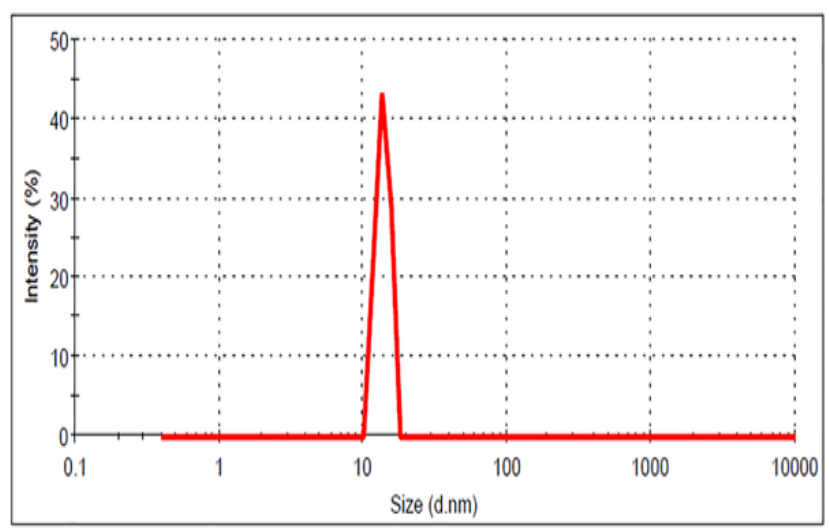

Figure 4: Globule size and size distribution observed for PrMIL based microemulsion.

\begin{tabular}{|c|c|c|c|c|c|c|c|c|}
\hline Formulations & Oil & TSM & $\begin{array}{l}\text { lonic } \\
\text { liquid }\end{array}$ & Water & $\begin{array}{l}\text { Globule } \\
\text { size }(n m)\end{array}$ & $\begin{array}{l}\text { Polydispersity } \\
\text { index }\end{array}$ & $\begin{array}{c}\text { Zeta } \\
\text { potential } \\
(\mathrm{mV})\end{array}$ & $\begin{array}{l}\text { Viscosity } \\
\left(\operatorname{cps} \times 10^{3}\right)\end{array}$ \\
\hline EmMILmicroemulsion(ME-1) & 60 & 25 & 15 & - & $164.3 \pm 3.3$ & $0.23 \pm 0.02$ & $11.3 \pm 0.7$ & $42.5 \pm 4.2$ \\
\hline PrMILmicroemulsion(ME-2) & 60 & 25 & 15 & - & $188.2 \pm 5.2$ & $0.15 \pm 0.03$ & $9.7 \pm 0.3$ & $34.33 \pm 4.5$ \\
\hline BuMILmicroemulsion(ME-3) & 60 & 25 & 15 & - & $174.1 \pm 4.2$ & $0.24 \pm 0.04$ & $12.8 \pm 0.7$ & $51.67 \pm 2.5$ \\
\hline EmIMILmicroemulsion(ME-4) & 60 & 25 & 15 & - & $179.3 \pm 2.8$ & $0.23 \pm 0.03$ & $10.5 \pm 0.1$ & $39.36 \pm 3.7$ \\
\hline o/w microemulsion(o/w ME) & 60 & 25 & - & 15 & $241.3 \pm 7.2$ & $0.20 \pm 0.07$ & $2.2 \pm 0.1$ & $24.11 \pm 2.8$ \\
\hline
\end{tabular}

The microemulsions loaded with $0.5 \%, 1 \%$ and $2 \% \mathrm{GMH}$. The globule size, polydispersity index, zeta potential and viscosity of selected microemulsion values are expressed as mean \pm SD $(n=3)$ 
conditions, showed no signed of instability. Particularly, no physical changes in colour or transparency were observed. Moreover, there were no significant $(p>0.05)$ differences observed in IL-microemulsion characteristics including mean globule sizes, polydispersity indices, zeta potential values and drug load assays.

Microemulsions are known to deliver drugs topically in deep tissue layers and reduce efficacy onset time in comparison to conventional formulations by altering the lipophilic nature of the tissue layers. ${ }^{19,20}$ The drug release properties of microemulsions have been associated with their physical characteristics, viscosity and constituents. ${ }^{21}$ As already described, we investigated GMH release through porcine vaginal tissue from several 1\% drug loaded IL-microemulsions (Figure 6). The cumulative drug release was evaluated for a period of $6 \mathrm{~h}$ as it corresponds to topical administration time. GMH release from a conventional 1\% drug loaded w/o microemulsion was studied as a control. It was observed that cumulative drug release from the conventional

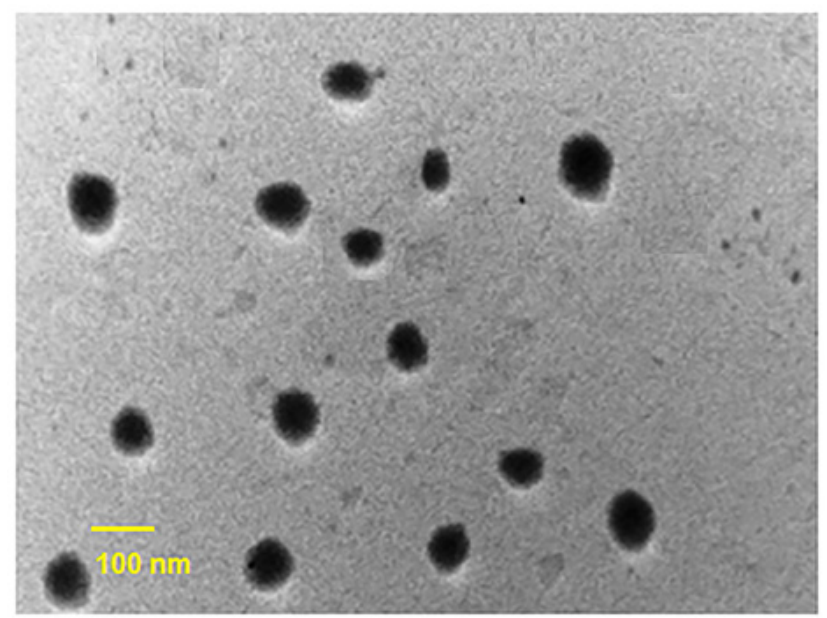

Figure 5: Transmission electron micrograph of PrMIL based microemulsion.

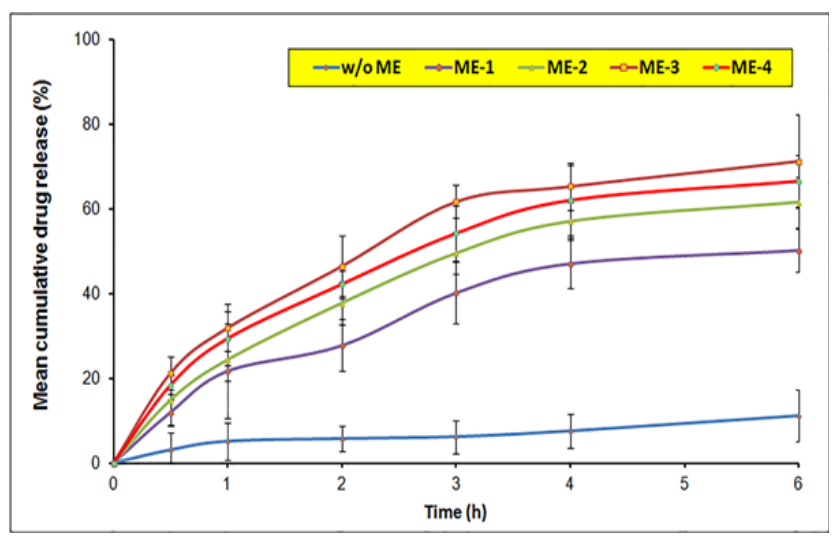

Figure 6: Ex vivo release profiles of prepared formulations. w/o microemulsion was very slow and massively incomplete $(11.19 \pm 3.81 \%$ in $6 \mathrm{~h})$ in comparison to IL-microemulsions. In contrast, the IL-microemulsions provided noticeably enhanced cumulative drug permeation. Particularly, with the IL-microemulsions, the percent drug permeation was found to be between $50.22 \pm 6.16 \%$ and $71.27 \pm 11.32 \%$, respectively. Upon comparing the results with the conventional w/o microemulsion, it was seen that the ionic liquid based microemulsions exhibited about 4.5 to 6.5 fold enhancement in $6 \mathrm{~h}$ cumulative drug permeation values. Also, when compared within the group, the drug permeation ability of the IL-microemulsions was observed to be in order of ME- $1<\mathrm{ME}-2<\mathrm{ME}-4<$ ME-3. The marginal lower permeation values of EtMIL and PrMIL ionic liquid based microemulsions, ME-1 and ME-2 could be attributed to the comparatively hydrophilic nature of constituent ionic liquids. The BuMIL provided maximum cumulative drug permeation values and therefore it provided to be most suitable for vaginal anticancer drug delivery.

Further, drug retention in tissue was evaluated after the completion of ex vivo permeation studies to gain insight on drug reservoir building ability of different formulations. Skin retention values are depicted in Figure 7. The conventional w/o microemulsion was only able to achieve a mean tissue retention value of $14.39 \pm 2.73 \mu \mathrm{g} / \mathrm{cm}^{2}$ for GMH. On the other hand, the IL-microemulsions provided approximately 3.1 to 3.6 times enhancements for mean tissue retention value of $14.39 \pm 2.73 \mu \mathrm{g} / \mathrm{cm}^{2}$ for $\mathrm{GMH}$, thereby providing a much improved drug reservoir, which could result in controlled release for more tie.

The cytotoxic potential of prepared IL-microemulsions was assessed using HeLa cervical cancer cell line as model cancer cells (Figure 8). The cells were incubated with $5 \mu \mathrm{L}$ IL-microemulsions loaded with varying

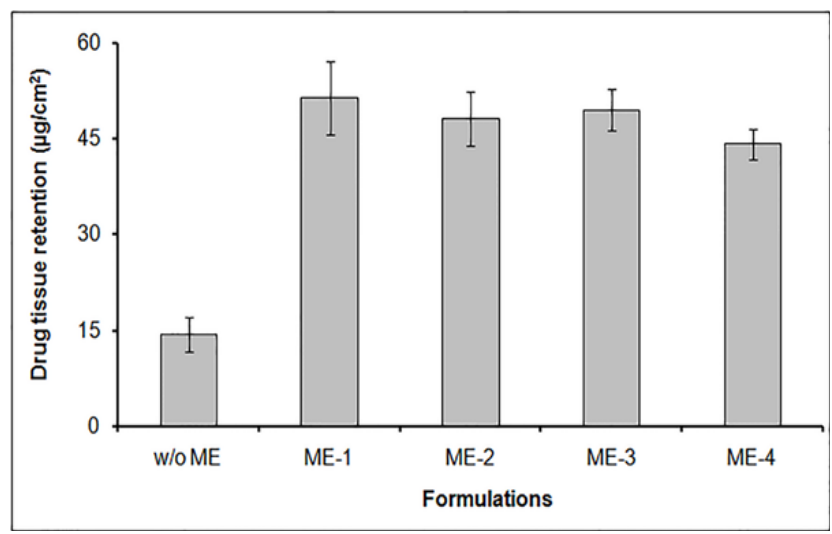

Figure 7: GMH retention achieved by prepared formulations in porcine vaginal tissue. 


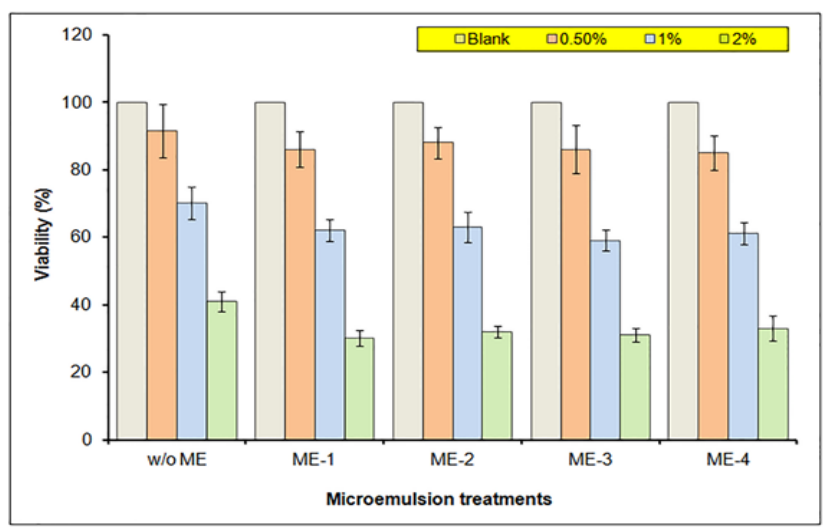

Figure 8: Dose dependent growth inhibition of human HeLa cells (human cervix carcinoma) studied using $0.5 \%, 1.0 \%$ and $2.0 \%$ of drug loaded formulations.

GMH drug content (namely, 0.5, 1 and \%) for 48 h. The results of cytotoxicity determinations showed that the GMH loaded IL-microemulsions exhibited noticeably improved cytotoxicity than conventional w/o drug loaded microemulsions.

\section{CONCLUSION}

This study attempted to developed novel ionic liquid based microemulsion systems of gemcitabine hydrochloride and evaluated the potential of vaginal delivery of GMH. The ionic liquids provided marked increase in drug solubility in comparison to water, thereby providing exceptional solubilizing compartment for proposed microemulsions. The prepared microemulsions were reported to possess the desirable physical properties and exhibited adequate 6-month stability. Also, excellent cumulative drug permeation and tissue retention were reported for the prepared formulations in our ex vivo studies in porcine vaginal tissues. We also observed the dosedependent in vitro cytotoxic effects in human HeLa cell lines. Although our studies reveal excellent therapeutic potential of ionic liquid based microemulsions, the studies are preliminary in natre, and the complete utility of these formulations could be only established after a combination of in vivo and in vitro studies. In this direction, we plan to conduct animal studies and cytotoxicity evaluations in many other cell lines. Our future findings in this direction will be published in due course of time.

\section{ACKNOWLEDGEMENT}

This study was supported by Zhejiang Medical and Health Research Project (No. 2017KY535).

\section{CONFLICT OF INTEREST}

The authors declare no conflict of interest.

\section{ABBREVIATIST}

GMH: Gemcitabine hydrochloride; IL: Ionic liquid; ME: Microemulsio1)

\section{REFERENCES}

1. Egorova KS, Ananikov VP. Biological activity of ionic liquids involving ionic and covalent binding: Tunable drug development platform. Encyclopedia of Ionic Liquids. Singapore: Springer Singapore. 2019;1-8.

2. Adawiyah N, Moniruzzaman $M$, Hawatulaila $S$, Goto $M$. Ionic liquids as a potential tool for drug delivery systems. Med Chem Comm. 2016;7(10):188197

3. Kandasamy $S$, Moniruzzaman M, Sivapragasam M, Shamsuddin MR, Mutalib MIA. Formulation and characterization of acetate based ionic liquid in oil microemulsion as a carrier for acyclovir and methotrexate. Separation and Purification Technology. 2018;196:149-56.

4. Kunz W, Zemb T, Harrar A. Using ionic liquids to formulate microemulsions: Current state of affairs. Current Opinion in Colloid and Interface Science. 2012;17(4):205-11.

5. Moniruzzaman M, Tamura M, Tahara $\mathrm{Y}$, Kamiya $\mathrm{N}$, et al. Ionic liquidin-oil microemulsion as a potential carrier of sparingly soluble drug: Characterization and cytotoxicity evaluation. International Journal of Pharmaceutics. 2010;400(1-2):243-50.

6. Bastiancich C, Bastiat G, Lagarce F. Gemcitabine and glioblastoma: Challenges and current perspectives. Drug Discovery Today. 2018;23(2):41623.

7. Shipley LA, Brown TJ, Cornpropst JD, Hamilton MA, Daniels WD, Culp HW. Metabolism and disposition of gemcitabine and oncolytic deoxycytidine analog, in mice, rats and dogs. Drug Metabolism and Disposition. 1992;20(6):849-55.

8. Kumar N, Jain R. Convenient syntheses of bulky group containing imidazolium ionic liquids. Journal of Heterocyclic Chemistry. 2011;49(2):370-4.

9. Kumar N, Goindi S, Kumar S, Jana AK. The effect of n-alkyl substituents on the usability of imidazolium cation-based ionic liquids in microemulsion systems: A technical note. AAPS Pharm Sci Tech. 2013;14(2):551-7.

10. Goindi S, Arora P, Kumar N, Puri A. Development of novel ionic liquid-based microemulsion formulation for dermal delivery of 5-Fluorouracil. AAPS Pharm Sci Tech. 2014;15(4):810-21.

11. Tsai YH, Hsieh YH, Huang YB, Chang JS, Huang CT, Wu PC. Microemulsions for intravesical delivery of gemcitabine. Chem Pharm Bull. 2010;58(11):14615

12. Hasan TN, Shafi G, Syed NA, Alfawaz MA, Alsaif MA, Munshi A, et al. Methanolic extract of Nigella sativa seed inhibits SiHa human cervical cancer cell proliferation through apoptosis. Nat Product Commun. 2010;58(11):1461-

13. Parsaee H, Asili J, Mousavi SH, Soofi H, Emami SA, Tayarani-Najaran Z. Apoptosis induction of Salvia chorassanica root extract on human cervical cancer cell line. Iran J Pharm Res. 2013;12(1):75-83.

14. Wang X, Xue M, Gu J, Fang X, Sha X. Transdermal microemulsion drug delivery system for impairing male reproductive toxicity and enhancing efficacy of Tripterygium wilfordii Hook f. Fitoterapia. 2012;83(4):690-8.

15. Xi J, Chang Q, Chan CK, Meng ZY, Wang GN, Sun JB, et al. Formulation development and bioavailability evaluation of a self-nanoemulsified drug delivery system of oleanolic acid. AAPS Pharm Sci Tech. 2009;10(1):172-82.

16. Pereira-Lachataignerais J, Pons R, Panizza P, Courbin L, Rouch J, Lopez O. Study and formation of vesicle systems with low polydispersed index by ultrasound method. Chem Phys Lipids. 2006;140(1-2):88-97.

17. Baspinar $\mathrm{Y}$, Borchert $\mathrm{HH}$. Penetration and release studies of positively and negatively charged nanoemulsions--is there a benefit of the positive charge? Int J Pharmacog. 2012;430(1-2):247-52. 
18. Peira E, Carlotti ME, Trotta C, Cavalli R, Trotta M. Positively charged microemulsions for topical application. Int J Pharm. 2008;346(1-2):119-23.
20. Kaushik D, Batheja P, Kilfoyle B, Rai V, Michiak-Kohn B. Percutaneous permeation modifiers: enhancement versus retardation. Expert Opin Drug Deliv. 2008;5(5):517-29.

21. Charro M, Vilas JM, Quintela JM, Guy R. Delivery of a hydrophilic solute through the skin from novel Microemulsion systems. Eur J Pharm Biopharm. $1997 ; 43(1): 37-42$

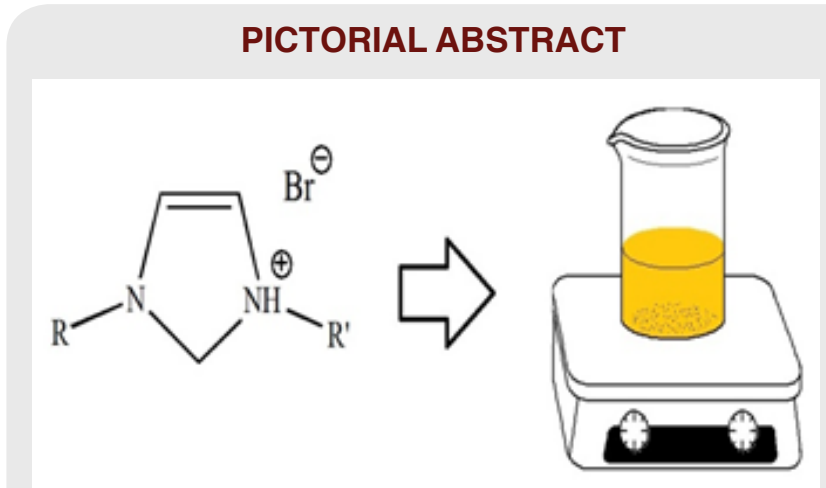

\section{SUMMARY}

We successfully devised novel ionic liquid based drug carriers for topical delivery of gemcitabine. The optimized formulations exhibited desirable physical and performance characteristics. Our studies however, need to be complemented with extensive animal studies for reinforcing the concept and potential of dermal delivery of gemcitabine using ionic liquid based carriers.

\section{About Authors}

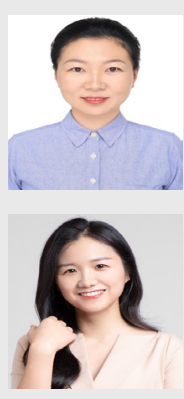

Juan Li is an Attending Physician in Women's Hospital, School of Medicine, Zhejiang University, China. From postgraduate till now, she has been engaged in clinical and basic research of tumor. She was also a partner in basic public welfare research program of Zhejiang Province in 2017.

Yuanyuan Chen is a Professor in the Department of Radiation Oncology, Affiliated Hangzhou Cancer Hospital, Zhejiang University, School of Medicine, China. She graduated from Medical College of Zhejiang University in 2010, and was honored to obtain Zhejiang Medical and Health Research Project in 2017. She is currently focusing on tumorigenesis and development in cervical cancer.

Cite this article: Chen Y, Li J. Development of a Novel lonic Liquid Based Microemulsion System for Gemcitabine Hydrochloride and in vitro Evaluation in Human Cervical Cancer HeLa Cells. Indian J of Pharmaceutical Education and Research. 2021;55(3):685-91. 